\title{
Adrenal vein sampling in primary aldosteronism: concordance of simultaneous vs sequential sampling
}

\author{
Mohamed-Karji Almarzooqi', Miguel Chagnon2, Gilles Soulez ${ }^{1,3}$, \\ Marie-France Giroux ${ }^{1}$, Patrick Gilbert ${ }^{1}$, Vincent L Oliva ${ }^{1}$, Pierre Perreault ${ }^{1}$, \\ Louis Bouchard', Isabelle Bourdeau', André Lacroix ${ }^{4}$ and Eric Therasse ${ }^{1}$ \\ 1'Department of Radiology, Centre Hospitalier de I'Université de Montréal (CHUM), Montréal, Quebec, \\ Canada, ${ }^{2}$ Department of Mathematics and Statistics, University of Montreal, Montréal, Quebec, Canada, \\ ${ }^{3}$ Centre de Recherche, CHUM (CRCHUM), Montréal, Quebec, Canada, and ${ }^{4}$ Department of Medicine, \\ Division of Endocrinology, Centre Hospitalier de I'Université de Montréal (CHUM), Montréal, \\ Quebec, Canada
}

\author{
Correspondence \\ should be addressed \\ to $\mathrm{E}$ Therasse \\ Email \\ eric.therasse.chum@ssss.gouv. \\ qc.ca
}

\section{Abstract}

Objective: Many investigators believe that basal adrenal venous sampling (AVS) should be done simultaneously, whereas others opt for sequential AVS for simplicity and reduced cost. This study aimed to evaluate the concordance of sequential and simultaneous AVS methods.

Design and methods: Between 1989 and 2015, bilateral simultaneous sets of basal AVS were obtained twice within $5 \mathrm{~min}$, in 188 consecutive patients ( 59 women and 129 men; mean age: 53.4 years). Selectivity was defined by adrenalto-peripheral cortisol ratio $\geq 2$, and lateralization was defined as an adrenal aldosterone-to-cortisol ratio $\geq 2$, the contralateral side. Sequential AVS was simulated using right sampling at $-5 \mathrm{~min}(t=-5)$ and left sampling at $0 \mathrm{~min}$ $(t=0)$.

Results: There was no significant difference in mean selectivity ratio $(P=0.12$ and $P=0.42$ for the right and left sides respectively) and in mean lateralization ratio $(P=0.93)$ between $t=-5$ and $t=0$. Kappa for selectivity between 2 simultaneous AVS was 0.71 (95\% Cl: $0.60-0.82)$, whereas it was 0.84 (95\% Cl: $0.76-0.92)$ and 0.85 (95\% Cl: $0.77-0.93$ ) between sequential and simultaneous AVS at respectively $-5 \mathrm{~min}$ and at $0 \mathrm{~min}$. Kappa for lateralization between 2 simultaneous AVS was $0.84(95 \% \mathrm{Cl}: 0.75-0.93)$, whereas it was $0.86(95 \% \mathrm{Cl}: 0.78-0.94)$ and 0.80 (95\% Cl: 0.71-0.90) between sequential AVS and simultaneous AVS at respectively $-5 \mathrm{~min}$ at $0 \mathrm{~min}$.

Conclusions: Concordance between simultaneous and sequential AVS was not different than that between 2 repeated simultaneous AVS in the same patient. Therefore, a better diagnostic performance is not a good argument to select the AVS method.

\section{Introduction}

Primary aldosteronism (PA) accounts for $5-10 \%$ of secondary hypertension and can often be surgically corrected $(1,2)$. Adrenal venous sampling (AVS) is the reference standard to differentiate unilateral adrenal aldosterone hypersecretion, mainly from aldosteroneproducing adenomas (APA), from bilateral adrenal
() 2017 European Society of Endocrinology Printed in Great Britain hyperplasia (BAH) in patients with PA $(3,4)$. This is especially important as APA can be treated with adrenalectomy, whereas treatment of BAH relies mainly on medication (5).

Although AVS is considered to be the reference standard for PA lateralization assessment, opinions differ 
in the literature on how the test should be performed technically (6). In sequential sampling, venous blood is drawn from the right and then from the left adrenal vein, whereas in simultaneous sampling, both adrenal veins are sampled at the same time with 2 catheters from 2 separate puncture sites (7). Worldwide, sequential sampling is performed by more centers than simultaneous sampling (8). Simultaneous AVS is more expensive and technically demanding than sequential $\operatorname{AVS}(6,7)$. The rationale behind simultaneous sampling is based on the assumption that right and left adrenal hormone secretion pulsatilities are correlated and, therefore, assessment of relative secretion of one adrenal in comparison to the other (lateralization of hormone secretion) needs to be done on sampling drawn at the same time $(3,7,9)$. Many believe that simultaneous blood sampling is likely to be crucial if AVS is performed without ACTH stimulation, and many centers use ACTH infusion to reduce stress-induced fluctuations in cortisol and aldosterone secretion during sequential AVS (3). However, ACTH's utility is limited by its confounding effect on the assessment of lateralization of aldosterone excess, and there is no conclusive evidence that ACTH-stimulated AVS leads to better outcomes $(3,10)$.

Whether the time required to position the catheter from one adrenal vein to the other is enough to change the diagnostic performance of AVS in the same patient is unknown and has never been ascertained (8). Our hypothesis is that the time lag between $2 \mathrm{AVS}$ in sequential mode is not enough to substantially change the result of basal AVS. The purpose of our study was to evaluate the concordance of sequential and simultaneous AVS.

\section{Subjects and methods}

\section{Study population}

All 198 consecutive patients referred to our center with biochemically proven PA who accepted unilateral adrenalectomy in the event that lateralization was confirmed and who were submitted to AVS between December 1989 and July 2015 were included. These patients, who underwent a total of 209 AVS sessions, were identified from an interventional radiology database, and their biochemical results were retrieved from the electronic health record of our medical center. So that all patients participate only once to the analyses, we included only the last AVS and excluded all prior AVS attempts in patients who had more than 1 AVS procedure (11 AVS procedures). AVS was repeated because of technical failure

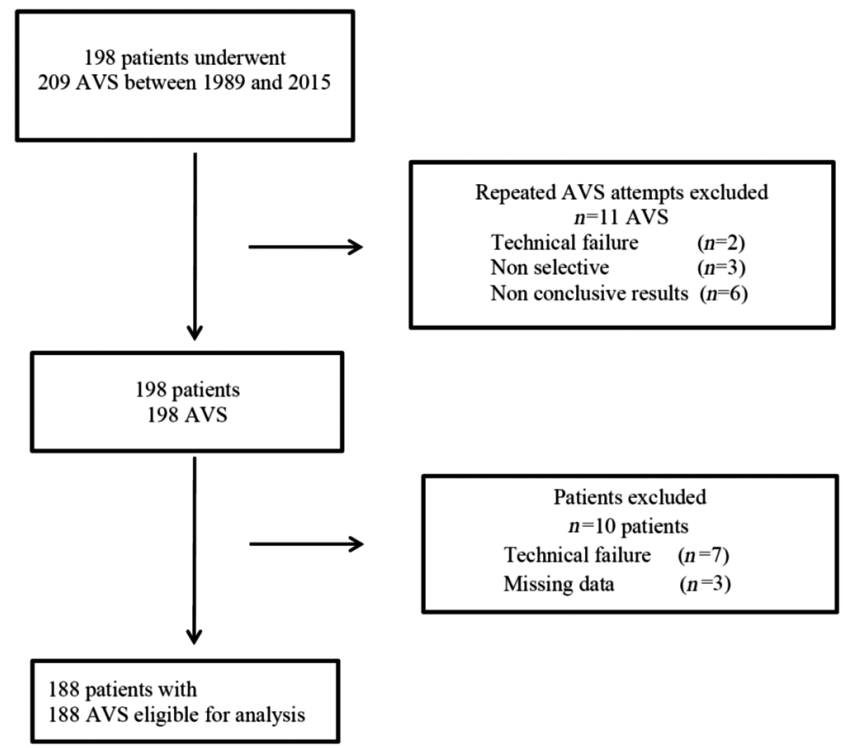

\section{Figure 1}

Flowchart of the study population. 209 adrenal venous sampling (AVS) studies were performed in 198 patients between 1989 and 2015. Repeated AVS attempts, technically unsuccessful AVS and AVS with missing data were excluded. Selectivity assessment was based on the remaining 188 patients, whereas lateralization concordance between sequential and simultaneous methods was assessed on 104 AVS that were bilaterally selective at both $t=-5 \mathrm{~min}$ and $t=0 \mathrm{~min}$.

(2 AVS), non-selective sampling on one or both sides (3 AVS) and unknown reasons (6 AVS). Ten patients were also excluded, either because AVSs were technical failures (7 patients) or because AVS data in patient files were missing (3 patients). Thus, 188 patients (59 women and 129 men; mean age $53.4 \pm 12.3$ years (range, $21-80$ years)) who underwent 188 AVS were included in the study analysis (Fig. 1). This patient population was assessed previously for other endpoints without overlapping the present study $(11,12,13)$. The criteria for primary aldosteronism diagnosis as well as patient preparation before AVS were published elsewhere $(11,12,13)$. Institutional Review Board approved this retrospective study and waived informed consent.

\section{AVS technique}

All 209 AVS sessions were performed by 6 fellowshiptrained interventional radiologists following identical methodology, with one of them (E.T.) undertaking 196 (94\%) of the procedures. The same AVS technique with the 
same protocol and blood sample timing was followed without modification for more than 25 years and has been described in detail $(11,12,13)$. Briefly, AVS was performed in the morning, between 08:00 and 12:00. A 6F sheath (Glidesheath, Terumo Interventional Systems) was inserted into the left common femoral vein for peripheral vein sampling. The left adrenal vein was catheterized with a $5 \mathrm{~F}$ Simmons medium or large curve catheter (Cordis, Miami, FL, USA), and the right adrenal vein was catheterized with a Chuang 2.5 catheter (Cook, Bloomington, IN, USA). The distal tip of a 0.014-inch guidewire (Transend, Boston Scientific, Freemont, CA, USA) was positioned in the $5 \mathrm{~F}$ catheter through a $\mathrm{Y}$ rotating hemostatic valve and advanced about $1-2 \mathrm{~cm}$ into the right adrenal vein to stabilize it and ease blood collection during AVS. Once inside the adrenal veins, the catheters were not moved from their positions between repeated sampling. Right and left adrenal and left iliac vein sampling were performed simultaneously $5 \mathrm{~min}(-5 \mathrm{~min})$ and immediately $(0 \mathrm{~min})$ before (basal AVS) a bolus injection of $250 \mu$ g cosyntropin (Cortrosyn, Organon Pharmaceuticals, West Orange, NJ, USA), and then repeated at 5, 10 and $15 \mathrm{~min}$. Clinical decision making toward or against adrenalectomy was based on the average of both basal samplings ( $t=-5 \mathrm{~min}$ and $t=0 \mathrm{~min}$ ) as well as on post-stimulation AVS performed after basal samplings. However, all analyses in this study are based only on basal AVS at $t=-5 \mathrm{~min}$ and $t=0 \mathrm{~min}$.

\section{Definitions}

Selectivity, i.e., adequate adrenal vein cannulation, was assessed by the adrenal-to-peripheral vein cortisol concentration ratio (selectivity index). Basal AVS was considered selective when selectivity index was $\geq 2$ as it is the most frequently used cut-off for basal AVS in the literature (8). Lateralization of aldosterone hypersecretion was assessed by the lateralization index (aldosteroneto-cortisol concentration ratio on the dominant side over aldosterone-to-cortisol concentration ratio on the contralateral side). Lateralization of aldosterone secretion was defined as a lateralization index $\geq 2$. Again, we adopted these cut-offs because they are the most frequently used for basal AVS in the literature (8).

In simultaneous AVS, both right and left AVS were performed at the same time. The sequential AVS method was simulated by using the right adrenal vein sampling at $-5 \mathrm{~min}$ and the left adrenal vein sampling at $0 \mathrm{~min}$. This sampling timing was selected because it represents how sequential sampling is usually done to decrease the time lag between sampling (adrenal vein cannulation is

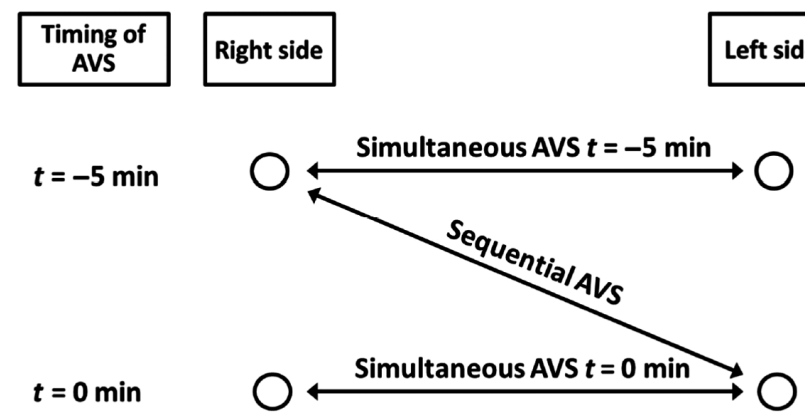

\section{Figure 2}

Schematic representation of simultaneous and sequential adrenal venous sampling (AVS). Blood samples were drawn simultaneously from both adrenal veins and the left iliac vein $5 \mathrm{~min}$ before ( $t=-5 \mathrm{~min}$ ) and immediately before ( $t=0 \mathrm{~min}$ ) intravenous bolus injection of $250 \mu \mathrm{g}$ cosyntropin. Sequential samplings were simulated by the use of peripheral and right adrenal hormone samplings at $t=-5 \mathrm{~min}$ and of peripheral and left adrenal hormone concentrations at $t=0 \mathrm{~min}$.

faster on the left than on the right side and, therefore, starting with right adrenal vein, cannulation would decrease the time lag between completion of right and left sampling). Figure 2 is a schematic representation of both simultaneous and sequential AVS.

\section{Comparison between sequential and simultaneous AVS}

A 2-step approach was taken to compare simultaneous to sequential AVS. First, we compared 2 repeated simultaneous AVS (at $t=-5 \mathrm{~min}$ and $t=0 \mathrm{~min}$ ) to assess the random variability of AVS selectivity and lateralization in the same patient and to ascertain whether there is a systematic variation of the AVS results with time. Then, we compared simultaneous to sequential AVS to evaluate whether selectivity and lateralization discordances between both methods were greater or similar to discordances between 2 repeated, simultaneous sampling.

We also assessed the correlation of adrenal aldosterone-to-cortisol ratio changes with time between the right and left sides because if these differences were correlated, sampling the right and left adrenal veins at different times would lead to time-dependent systematic error in sequential sampling.

Analyses of selectivity involved all AVS included in this study and analyses of lateralization were limited to a subgroup of bilaterally selective AVS (selectivity index $\geq 2$ ). 


\section{Statistical analysis}

Comparison of selectivity and lateralization between 2 repeated simultaneous AVS and between simultaneous and sequential AVS was analyzed as continuous (selectivity and lateralization index) and categorical (selectivity and lateralization index $\geq 2$ ) variables. Differences in continuous ratio between 2 simultaneous AVS performed at $t=-5 \mathrm{~min}$ and at $t=0 \mathrm{~min}$ were assessed after $\log$ transformation because of their skewed distribution. Logtransformed values at $t=-5$ and $t=0$ were compared by paired $t$-test. Same approach was used for lateralization, on selective subjects only, and agreement was measured on log-log scale using intraclass correlation coefficient. Agreement for selectivity and lateralization status of AVS were studied using a 95\% confidence interval of concordance and Kappa statistics. Agreement of selectivity and lateralization with time were also described as a percentage change with Bland-Altman approach on logarithm scale. Descriptive statistics are reported as mean \pm S.D., or frequency and percentage, as appropriate. Statistical analyses were performed by a biostatistician with IBM SPSS statistics, version 23 with a significance level of $5 \%$.

\section{Results}

\section{Selectivity and lateralization ratio}

Table 1 reports the mean differences of logtransformed selectivity and lateralization ratios between simultaneous AVS performed at $t=-5$ and $t=0 \mathrm{~min}$, whereas Supplementary Table 1 (see section on supplementary data given at the end of this article) reports the original data before log transformation. For both ratios, there were no significant differences during the 5-min period. Bland-Altman analysis of selectivity

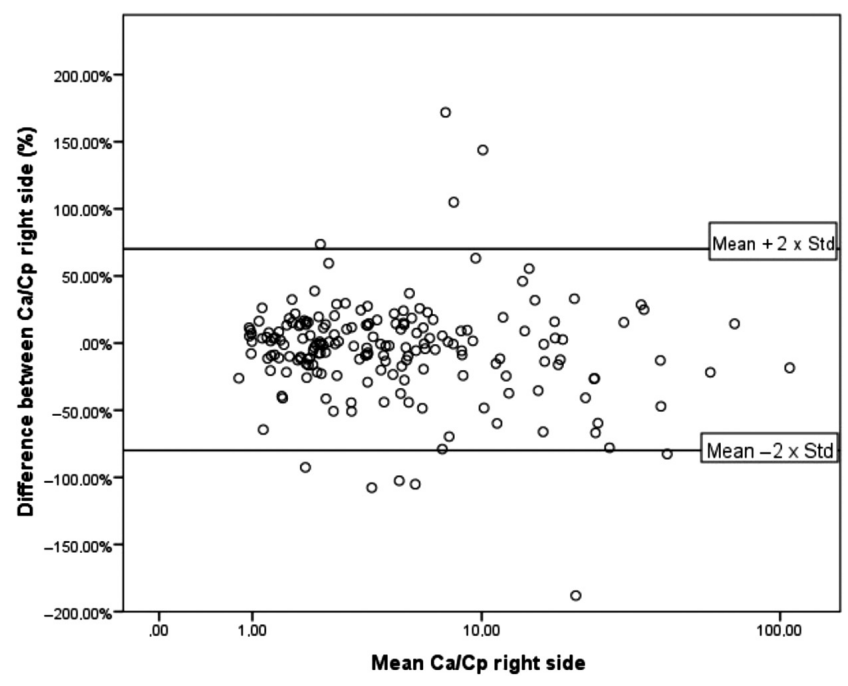

Figure 3

Bland-Altman analysis of selectivity ratio between $t=-5 \mathrm{~min}$ and $t=0 \mathrm{~min}$ for the right adrenal venous sampling. There is no systematic difference between $t=-5 \mathrm{~min}$ and $t=0 \mathrm{~min}$, but selectivity ratio variability with time is substantial.

ratio for the right (Fig. 3) and left side (Fig. 4), as well as for the lateralization ratio (Fig. 5) shows no systematic difference between $t=-5 \mathrm{~min}$ and $t=0 \mathrm{~min}$. However, they also demonstrate an important variability of selectivity and lateralization ratios between the 2 sampling.

Figure 6 reports the correlation of lateralization ratio between $t=-5 \mathrm{~min}$ and $t=0 \mathrm{~min}$. In addition to the important lateralization ratio variability, this graph demonstrates its effect on lateralization status with a cut-off value $\geq 2$. Figure 7 shows the correlation between right and left adrenal aldosterone-to-cortisol ratio changes between the $t=-5$ and $t=0$ time points. There was almost no correlation of adrenal aldosterone-tocortisol ratio changes with time between the right and

Table 1 Differences in adrenal hormone concentrations ratios between simultaneous AVS performed at $t=-5 \mathrm{~min}$ and $t=0 \mathrm{~min}$ between right and left AVS.

\begin{tabular}{|c|c|c|c|c|c|}
\hline & n & $t=-5$ & $t=0$ & $\begin{array}{l}\text { Differences between } \\
t=-5 \mathrm{~min} \text { and } t=0 \mathrm{~min}\end{array}$ & $P$ Value \\
\hline \multicolumn{6}{|l|}{ Mean log selectivity index } \\
\hline Right side & 187 & $0.643 \pm 0.451$ & $0.620 \pm 0.432$ & $-0.0234 \pm 0.205$ & 0.120 \\
\hline Left side & 186 & $0.614 \pm 0.421$ & $0.626 \pm 0.432$ & $0.0116 \pm 0.195$ & 0.419 \\
\hline \multicolumn{6}{|l|}{ Mean $\log A / C$} \\
\hline Right side & 187 & $0.812 \pm 0.671$ & $0.805 \pm 0.679$ & $-0.00693 \pm 0.299$ & 0.751 \\
\hline Left side & 186 & $0.799 \pm 0.648$ & $0.797 \pm 0.645$ & $-0.00222 \pm 0.268$ & 0.910 \\
\hline Mean log lateralization index & 104 & $0.284 \pm 1.05$ & $0.281 \pm 1.09$ & $0.00275 \pm 0.336$ & 0.934 \\
\hline
\end{tabular}

$t=-5$, sampling at $-5 \mathrm{~min} ; t=0$, sampling at $0 \mathrm{~min}$; Selectivity index, adrenal over peripheral venous cortisol ratio; $\mathrm{A} / \mathrm{C}$, adrenal vein aldosterone-tocortisol ratio; AVS, adrenal venous sampling. 


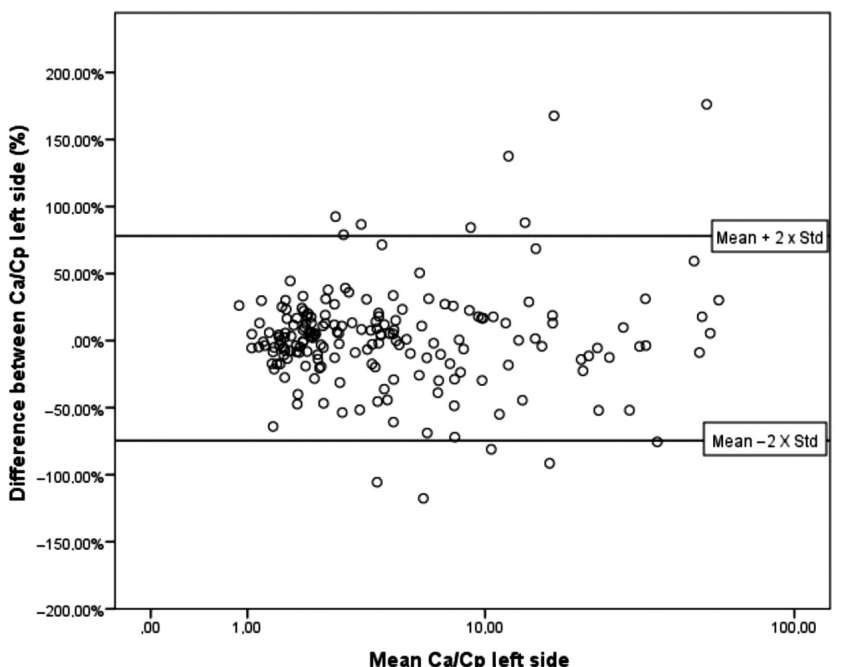

Figure 4

Bland-Altman analysis of selectivity ratio between $t=-5 \mathrm{~min}$ and $t=0 \mathrm{~min}$ for the left adrenal venous sampling. There is no systematic difference between $t=-5 \mathrm{~min}$ and $t=0 \mathrm{~min}$, but selectivity ratio variability with time is substantial.

left sides $\left(R^{2}=0.053\right)$ and, therefore, no systematic error should result from performing right and left sampling at different times.

Table 2 compares mean log lateralization ratios according to AVS methods (simultaneous and sequential) and shows the ratio correlation between 2 simultaneous

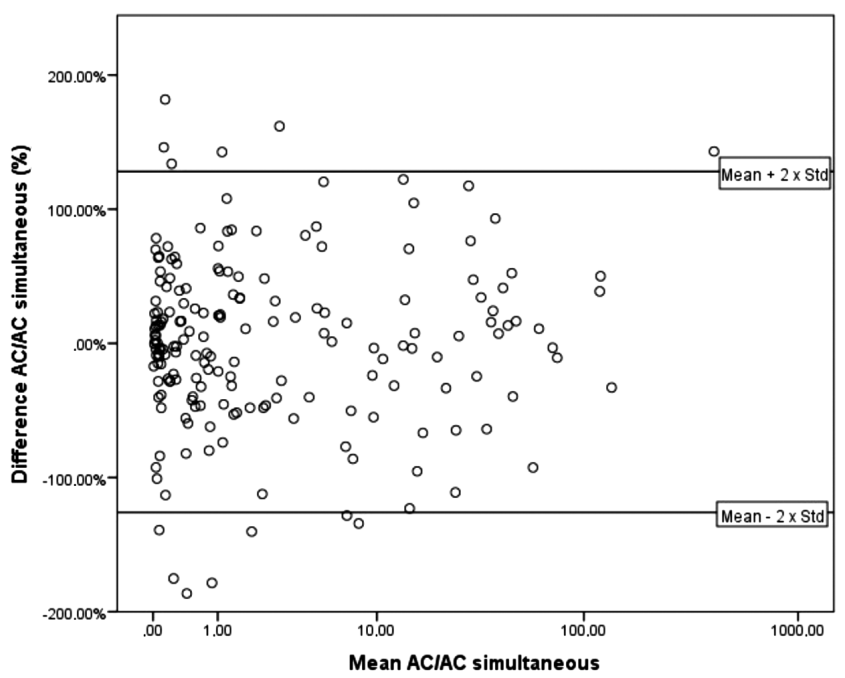

Figure 5

Bland-Altman analysis of lateralization ratio between $t=-5 \mathrm{~min}$ and $t=0 \mathrm{~min}$. Although there is no systematic difference between $t=-5 \mathrm{~min}$ and $t=0 \mathrm{~min}$, lateralization ratio variability with time is substantial.

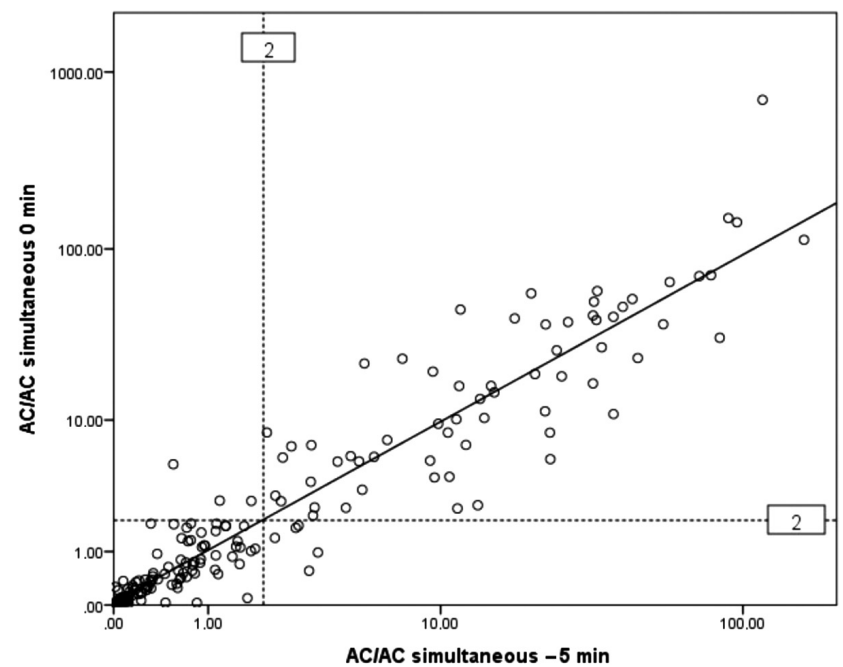

Figure 6

Scatterplot showing correlation of lateralization ratios between adrenal venous sampling at $t=-5 \mathrm{~min}$ and at $t=0 \mathrm{~min}$. Pearson correlation for log lateralization ratio was 0.946 .

AVS and between simultaneous and sequential AVS. Supplementary Table 2 reports the original data before $\log$ transformation. No significant differences in mean log lateralization ratio were apparent with all comparisons. Correlation of lateralization ratios between

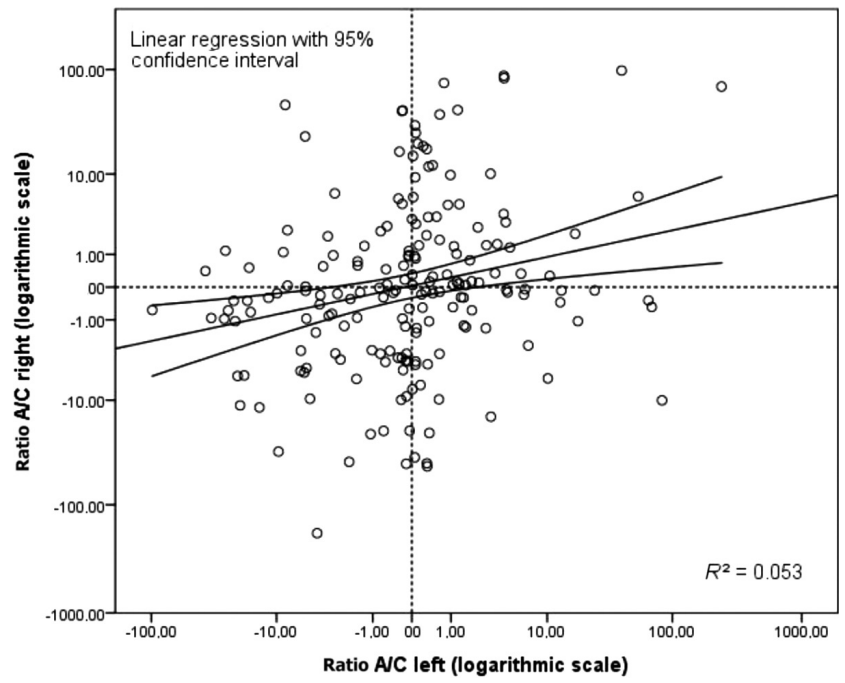

Figure 7

Correlation between right and left aldosterone/cortisol (A/C) changes with time. Correlation of $A / C$ changes with time between the right and the left side is very poor $\left(R^{2}=0.053\right)$, and right and left adrenal $A / C$ ratio changes with time are almost completely independent. 
Table 2 Correlation of lateralization ratios between simultaneous and sequential AVS in subjects in whom AVS was bilaterally selective.

\begin{tabular}{|c|c|c|c|c|c|c|c|}
\hline AVS methods & $n$ & $\begin{array}{c}\text { Mean log } \\
\text { lateralization index }\end{array}$ & $\begin{array}{l}\text { AVS method for } \\
\text { comparison }\end{array}$ & $\begin{array}{c}\text { Mean log } \\
\text { lateralization index }\end{array}$ & Mean difference & P Value & ICC $(95 \% \mathrm{Cl})$ \\
\hline $\begin{array}{l}\text { Simultaneous } \\
t=-5\end{array}$ & 104 & $0.284 \pm 1.05$ & Simultaneous $t=0$ & $0.281 \pm 1.09$ & $0.00275 \pm 0.336$ & 0.93 & $0.946(0.929 ; 0.959)$ \\
\hline Sequential & 110 & & & & $-0.00019 \pm$ & & \\
\hline Sequential & 111 & $0.245 \pm 1.09$ & Simultaneous $t=-5$ & $0.268 \pm 1.04$ & $0.0229 \pm 0.270$ & 0.37 & $0.967(0.956 ; 0.975)$ \\
\hline
\end{tabular}

$t=-5$, sampling at $-5 \mathrm{~min} ; t=0$, sampling at $0 \mathrm{~min}$; AVS, adrenal venous sampling; ICC, intraclass correlation coefficient; Simultaneous, right and left adrenal venous sampling at the same time; Sequential, right and left adrenal venous sampling at $-5 \mathrm{~min}$ and $0 \mathrm{~min}$ respectively; $95 \% \mathrm{Cl}$, $95 \%$ confidence interval.

2 simultaneous AVS was good but not better than that between simultaneous and sequential AVS.

Given that the cortisol/aldosterone assay changes over the 25 years of data collection could potentially affect the result of the study, we assessed the variability of both selectivity and lateralization index over time by comparing the first and second half of the study period. These comparisons demonstrated no significant difference of mean selectivity and lateralization index over time.

\section{Selectivity and lateralization status (cut-off ratio $\geq 2$ )}

Table 3 shows concordance of selectivity status between simultaneous and sequential AVS with selectivity index cut-off $\geq 2$. There were no significant differences in concordance for all comparisons. Concordance between 2 simultaneous AVS was not better than that between simultaneous and sequential AVS. Discordances of selectivity status were $13.5 \%(25 / 185)$ between 2 simultaneous AVS obtained with a 5-min interval.
With selectivity ratio cut-off $\geq 2,104 / 188$ (55.3\%) AVS were selective bilaterally at both $t=-5 \mathrm{~min}$ and $t=0 \mathrm{~min}$. Table 4 shows concordance of lateralization status between simultaneous and sequential AVS for lateralization index $\geq 2$. Differences in concordance were not significant for all comparisons, and concordance between 2 simultaneous AVS was not better than that between simultaneous and sequential AVS. Discordances of lateralization status were 10.6\% (11/104) between 2 simultaneous AVS obtained at 5-min interval.

Increasing the selectivity index cut-off from $\geq 2$ to $\geq 3$ improved the concordance between $t=-5$ and $t=0 \mathrm{~min}$ from $86.5 \%$ to $91.4 \%(169 / 185)$ and Kappa from 0.71 to $0.83(0.75 ; 0.91 ; P=0.50)$, whereas increasing the lateralization cut-off ratio from $\geq 2$ to $\geq 4$ improved the concordance from $89.4 \%$ to $92.3 \%$ (96/104) and Kappa from 0.84 to 0.88 ( $0.80 ; 0.96 ; P=0.74)$. However, these differences are not statistically significant and increasing selectivity and lateralization cut-offs lead respectively to an increasing number of non-selective (from 36.8\% to $53.5 \%$ ) and non-lateralized AVSs (from $24.5 \%$ to $37.5 \%$ ).

Table 3 Concordance of bilateral selectivity index $\geq 2$ between simultaneous and sequential AVS.

\begin{tabular}{|c|c|c|c|c|}
\hline \multirow[b]{2}{*}{ Bilateral selectivity } & \multicolumn{2}{|c|}{ Bilateral selectivity* } & \multirow[b]{2}{*}{ Concordance $(95 \% \mathrm{Cl})^{\dagger}$} & \multirow[b]{2}{*}{ Kappa* $(95 \% \mathrm{Cl})$} \\
\hline & Yes & No & & \\
\hline Simultaneous $t=0$ & Simultaneous $t=-5$ & & $86.5 \%(80.6 \% ; 91.0 \%)$ & $0.71(0.60 ; 0.82)$ \\
\hline Yes & $56.2 \%(104)$ & $5.9 \%(11)$ & & \\
\hline No & $7.6 \%(14)$ & $30.3 \%(56)$ & & \\
\hline Sequential & Simultaneous $t=-5$ & & $92.5 \%(87.4 \% ; 95.7 \%)$ & $0.84(0.76 ; 0.92)$ \\
\hline Yes & $59.7 \%(111)$ & $3.8 \%(7)$ & & \\
\hline No & $3.8 \%(7)$ & $32.8 \%(61)$ & & \\
\hline Sequential & Simultaneous $t=0$ & & $93.0 \%(88.1 \% ; 96.1)$ & $0.85(0.77 ; 0.93)$ \\
\hline Yes & $59.1 \%(110)$ & $4.3 \%(8)$ & & \\
\hline No & $2.7 \%(5)$ & $33.9 \%(63)$ & & \\
\hline
\end{tabular}

$t=-5$, sampling at $-5 \mathrm{~min} ; t=0$, sampling at $0 \mathrm{~min}$; AVS, adrenal venous sampling; Simultaneous, right and left adrenal venous sampling performed at the same time; Sequential, right sampling at $t=-5 \mathrm{~min}$ and left sampling at $0 \mathrm{~min}$; Selectivity, yes if selectivity index $\geq 2$, and no if selectivity index $<2$; $95 \% \mathrm{Cl}, 95 \%$ confidence interval.

${ }^{*}$ Categorical data are given as percentages, with numbers of AVS in parentheses. ${ }^{\dagger}$ Values in parentheses=interquartile range. 
Table 4 Concordance of lateralization status between simultaneous and sequential AVS on subjects in whom AVS was bilaterally selective at both $t=-5$ and $t=0 \mathrm{~min}$.

\begin{tabular}{|c|c|c|c|c|c|}
\hline \multirow[b]{2}{*}{ Lateralization } & \multicolumn{3}{|c|}{ Lateralization* } & \multirow[b]{2}{*}{ Concordance $(95 \% \mathrm{Cl})^{\dagger}$} & \multirow[b]{2}{*}{ Kappa $(95 \% \mathrm{Cl})^{\dagger}$} \\
\hline & Right & None & Left & & \\
\hline Simultaneous $t=0$ & \multicolumn{3}{|c|}{ Simultaneous AVS $t=-5$} & $89.4 \%(81.5 \% ; 94.3)$ & $0.84(0.75 ; 0.93)$ \\
\hline Right & $46(44.2 \%)$ & $2(1.9 \%)$ & $0(0.0 \%)$ & & \\
\hline None & $2(1.9 \%)$ & $20(19.2 \%)$ & $1(1.0 \%)$ & & \\
\hline Left & $0(0.0 \%)$ & $6(5.8 \%)$ & $27(26.0 \%)$ & & \\
\hline Sequential & \multicolumn{3}{|c|}{ Simultaneous AVS $t=-5$} & $91.0 \%(83.8 \% ; 95.7 \%)$ & $0.86(0.78 ; 0.94)$ \\
\hline Right & $49(44.1 \%)$ & $1(0.9 \%)$ & $0(0.0 \%)$ & & \\
\hline None & $2(1.8 \%)$ & $23(20.7 \%)$ & $1(0.9 \%)$ & & \\
\hline Left & $0(0.0 \%)$ & $6(5.4 \%)$ & $29(26.1 \%)$ & & \\
\hline Sequential & \multicolumn{3}{|c|}{ Simultaneous AVS $t=0$} & $87.3 \%(79.2 \% ; 92.9 \%)$ & $0.80(0.71 ; 0.90)$ \\
\hline Right & $46(41.8 \%)$ & $2(1.8 \%)$ & $0(0.0 \%)$ & & \\
\hline None & $2(1.8 \%)$ & $20(18.2 \%)$ & $6(5.5 \%)$ & & \\
\hline Left & $1(0.9 \%)$ & $3(2.7 \%)$ & $30(27.3 \%)$ & & \\
\hline
\end{tabular}

$t=-5$, sampling at $-5 \mathrm{~min} ; t=0$, sampling at $0 \mathrm{~min}$; AVS, adrenal venous sampling; $95 \% \mathrm{Cl}, 95 \%$ confidence interval; Simultaneous, right and left adrenal venous sampling performed at the same time; Sequential, right and left adrenal venous sampling at $-5 \mathrm{~min}$ and $0 \mathrm{~min}$ respectively.

*Categorical data are given as percentages, with numbers of AVS in parentheses. ${ }^{+}$Values in parentheses=interquartile range.

\section{Discussion}

Our study demonstrates that simultaneous and sequential AVS yielded similar results in terms of selectivity and lateralization as long as right and left sampling were obtained within $5 \mathrm{~min}$. There was no significant difference in mean selectivity and lateralization ratios between 2 AVS performed within $5 \mathrm{~min}$, and there was no significant difference in concordance between 2 simultaneous AVS and between a simultaneous and a sequential AVS. These results indicate no systematic, time-dependent bias when sampling was not performed at the same time. However, our data also show substantial intra-patient variations in selectivity and lateralization ratios between 2 repeated, simultaneous AVS performed within $5 \mathrm{~min}$, with a discordance rate of $13.5 \%$ for selectivity and of $10.6 \%$ for lateralization.

Substantial intra-patient selectivity and lateralization ratio variations without significant change in mean values with time suggest that variability of repeated AVS results in our study was mostly due to random rather than systematic, time-dependent error (14). Poor correlation between right and left side variations of adrenal aldosterone-to-cortisol ratio with time also support random rather than systematic errors as the source of variability with time. Such random errors are responsible not only for discordances between 2 simultaneous AVS but also for discordances between simultaneous and sequential AVS. Random variability of hormone secretion with time was probably due to multifactorial causes such as independent right and left adrenal hormonal secretion pulsatilities, various degrees of catheter selectivity (catheter shift with respiratory movements) and laboratory errors. These sources of random variability may explain the discordances in selectivity and lateralization between 2 simultaneous AVS performed 5-min apart. On the other hand, the absence of systematic variations with time could explain why mean selectivity and lateralization ratio were similar and why concordance rate between simultaneous and sequential AVS was not significantly different.

Our results demonstrate that prevention of a timedependent hormone sampling bias is not a good argument to select simultaneous over sequential sampling. As simultaneous AVS is more invasive, requiring the insertion of 2 catheters, is more time consuming and more expensive than sequential AVS, centers that implement basal AVS could preferentially opt for the sequential technique.

Although simultaneous AVS is frequently performed without stimulation, ACTH perfusion is generally suggested during sequential AVS to reduce stress-induced fluctuations in cortisol and aldosterone secretion with time (3). However, given the absence of systematic change in selectivity and lateralization between repeated AVS performed within $5 \mathrm{~min}$, it is uncertain that ACTH infusion would benefit more to one of these AVS methods. Although ACTH also improves selectivity detection, this advantage is less important nowadays given the recently described techniques to identify adequate adrenal vein catheterization, such as preplanning CT, cone-beam CT, real-time cortisol assays and the adrenal-to-peripheral aldosterone ratio for efficient proofs of successful adrenal catheterization $(4,12,15,16,17)$.

Variability of AVS results is a substantial limitation of this diagnostic test. As most variability of 2 AVS 
measurements within $5 \mathrm{~min}$ is attributed to random rather than systematic error, averaging multiple measurements could be a way to improve fidelity of the AVS results (14). Although collecting multiple measurements is difficult with the sequential method, it is relatively easy with the simultaneous approach. Hence, a potential advantage of simultaneous over sequential AVS could be the ability to undertake repeated sampling over time to average AVS hormone concentrations and ratios.

Our study has some limitations. First, our results do not apply to centers administering ACTH bolus injection because, contrary to steady state perfusion, it will quickly and systematically increase adrenal hormone secretion bilaterally with time. Therefore, a sequential AVS after ACTH bolus injection would lead to systematic timedependent sampling bias. Hence, for centers implementing the post-ACTH bolus (instead of a continuous infusion) AVS protocol, simultaneous sampling would still be preferable.

Second, comparisons of selectivity and lateralization between sequential and simultaneous AVS may not apply if the time between right and left AVS is beyond $5 \mathrm{~min}$, the lag which was tested in our study. In contrast to our results, a previous study reported significant selectivity ratio changes that could have been due to stress reaction related to the intervention and environment in repeated AVS after 15 min (9). However, even in that study, decline of the selectivity ratio with time did not affect the AVS proportion that was held to be selective with cut-offs ranging from 1.10 to 2.00 (9). Although selectivity discrepancies were greater for sequential AVS in the study by Seccia et al. (9), they mainly resulted from the calculation of selectivity ratios using adrenal and peripheral samples collected at different times. For sequential sampling, it is rather recommended to take a sample for cortisol and aldosterone assay from a peripheral vein every time an adrenal vein is sampled (6). In addition, in sequential catheterization, the mean time needed to catheterize the left adrenal vein after right adrenal vein catheterization and sampling was reported to be $3.7 \mathrm{~min}$, and we believe that it should take less than $5 \mathrm{~min}$ in the vast majority of cases (4).

Third, in true sequential AVS, the right is usually cannulated first, and then the catheter was removed and a different catheter was inserted to cannulate the left. Although unlikely in experienced hands, it is possible that manipulation of catheters after collection of the right samples could lead to the stimulation of steroid synthesis via ACTH. Finally, we cannot rule out small differences in selectivity and lateralization between sequential and simultaneous AVS given the sample size of our study.

Finally, another limitation of this study is the observational character of the study with retrospective data analysis over a 25-year period. However, there were no variations in the AVS protocol over the span of the study period.

In conclusion, concordance between sequential and simultaneous AVS is similar to the concordance between 2 repeated simultaneous AVS in the same patient and, therefore, both approaches could be used interchangeably. However, our study also demonstrated substantial discordances in selectivity and lateralization between 2 repeated AVS performed within $5 \mathrm{~min}$ in the same patient. These discordances appear to be mainly due to random rather than systematic time-dependent errors and, therefore, could potentially be improved by averaging multiple AVS results.

\section{Supplementary data}

This is linked to the online version of the paper at http://dx.doi.org/10.1530/ EJE-16-0701.

\section{Declaration of interest}

The authors declare that there is no conflict of interest that could be perceived as prejudicing the impartiality of the research reported.

\section{Funding}

This research did not receive any specific grant from any funding agency in the public, commercial or not-for-profit sector.

\section{References}

1 Rossi GP, Bernini G, Caliumi C, Desideri G, Fabris B, Ferri C, Ganzaroli C, Giacchetti G, Letizia C, Maccario M et al. A prospective study of the prevalence of primary aldosteronism in 1125 hypertensive patients. Journal of the American College of Cardiology 200648 2293-2300. (doi:10.1016/j.jacc.2006.07.059)

2 Douma S, Petidis K, Doumas M, Papaefthimiou P, Triantafyllou A, Kartali N, Papadopoulos N, Vogiatzis K \& Zamboulis C. Prevalence of primary hyperaldosteronism in resistant hypertension: a retrospective observational study. Lancet 2008371 1921-1926. (doi:10.1016/S01406736(08)60834-X)

3 Rossi GP, Auchus RJ, Brown M, Lenders JW, Naruse M, Plouin PF, Satoh F \& Young WF Jr. An expert consensus statement on use of adrenal vein sampling for the subtyping of primary aldosteronism. Hypertension 201463 151-160. (doi:10.1161/ HYPERTENSIONAHA.113.02097)

4 Sacks BA, Brook OR \& Brennan IM. Adrenal venous sampling: promises and pitfalls. Current Opinion in Endocrinology, Diabetes, and Obesity 201320 180-185. (doi:10.1097/MED.0b013e32835f26c9)

5 Rossi G. Diagnosis and treatment of primary aldosteronism. Reviews in Endocrine and Metabolic Disorders 201112 27-36. (doi:10.1007/s11154011-9162-8) 
6 Monticone S, Viola A, Rossato D, Veglio F, Reincke M, GomezSanchez C \& Mulatero P. Adrenal vein sampling in primary aldosteronism: towards a standardised protocol. Lancet Diabetes and Endocrinology 20153 296-303. (doi:10.1016/S2213-8587(14)70069-5)

7 Carr CE, Cope C, Cohen DL, Fraker DL \& Trerotola SO. Comparison of sequential versus simultaneous methods of adrenal venous sampling. Journal of Vascular and Interventional Radiology $2004 \mathbf{1 5}$ 1245-1250. (doi:10.1097/01.RVI.0000134495.26900.6A)

8 Rossi GP, Barisa M, Allolio B, Auchus RJ, Amar L, Cohen D, Degenhart C, Deinum J, Fischer E, Gordon R et al. The Adrenal Vein Sampling International Study (AVIS) for identifying the major subtypes of primary aldosteronism. Journal of Clinical Endocrinology and Metabolism 201297 1606-1614. (doi:10.1210/jc.2011-2830)

9 Seccia TM, Miotto D, Battistel M, Motta R, Barisa M, Maniero C, Pessina AC \& Rossi GP. A stress reaction affects assessment of selectivity of adrenal venous sampling and of lateralization of aldosterone excess in primary aldosteronism. European Journal of Endocrinology 2012166 869-875. (doi:10.1530/EJE-11-0972)

10 Seccia TM, Miotto D, De Toni R, Pitter G, Mantero F, Pessina AC \& Rossi GP. Adrenocorticotropic hormone stimulation during adrenal vein sampling for identifying surgically curable subtypes of primary aldosteronism: comparison of 3 different protocols. Hypertension 2009 53 761-766. (doi:10.1161/HYPERTENSIONAHA.108.128553)

11 Kaitoukov Y, Soulez G, Oliva VL, Giroux MF, Bourdeau I, Lacroix A, Gilbert P \& Therasse E. Coaxial guide wire placement in the right adrenal vein for repeated adrenal venous samplings. Cardiovascular and Interventional Radiology 201437 795-799. (doi:10.1007/s00270013-0794-9)
12 Mailhot JP, Traistaru M, Soulez G, Ladouceur M, Giroux MF, Gilbert P, Zhu PS, Bourdeau I, Oliva VL, Lacroix A et al. Adrenal vein sampling in primary aldosteronism: sensitivity and specificity of basal adrenal vein to peripheral vein cortisol and aldosterone ratios to confirm catheterization of the adrenal vein. Radiology 2015277 887-894. (doi:10.1148/radiol.2015142413)

13 El Ghorayeb N, Mazzuco TL, Bourdeau I, Mailhot JP, Zhu PS, Thérasse E \& Lacroix A. Basal and post ACTH aldosterone and its ratios are useful during adrenal vein sampling in primary aldosteronism. Journal of Clinical Endocrinology and Metabolism 2016 101 1826-1835. (doi:10.1210/jc.2015-3915)

14 Taylor JR. An Introduction to Error Analysis: The Study of Uncertainties in Physical Measurements, 2nd edition, pp 94-97. Chapter 4.1: Random and Systematic Errors. Mill Valley, CA, USA: University Science Books, 1997.

15 Busser WM, Arntz MJ, Jenniskens SF, Deinum J, Hoogeveen YL, de Lange F \& Schultze Kool LJ. Image registration of cone-beam computer tomography and preprocedural computer tomography aids in localization of adrenal veins and decreasing radiation dose in adrenal vein sampling. Cardiovascular and Interventional Radiology 201538 993-977. (doi:10.1007/s00270-014-0969-z)

16 Stowasser M. Improving the success and reliability of adrenal venous sampling: focus on intraprocedural cortisol measurement. Clinical Chemistry 201258 1275-1277. (doi:10.1373/clinchem.2012.182469)

17 Morita S, Nishina Y, Yamazaki H, Sonoyama Y, Ichihara A \& Sakai S. Dual adrenal venous phase contrast-enhanced MDCT for visualization of right adrenal veins in patients with primary aldosteronism. European Radiology 201626 2073-2077. (doi:10.1007/s00330-015-4073-9)

Received 17 August 2016

Revised version received 21 October 2016

Accepted 11 November 2016 